\title{
1. Departmontof
}

Journal of Rehabilitation Research

and Development Vol. 28 No. 4, 1991

Pages 33-38

\section{Thirty-minute continuous sitting force measurements with differ- ent support surfaces in the spinal cord injured and able-bodied}

\author{
Paul Gilsdorf, BS; Robert Patterson, PhD; Steven Fisher, MD \\ University of Minnesota, Minneapolis, MN 55455; Department of Physical Medicine and Rehabilitation, St. Paul \\ Ramsey Medical Center, St. Paul, MN 55110
}

\begin{abstract}
Able-bodied, paraplegic, and quadriplegic subjects sat for 30-minute intervals on various surfaces in a wheelchair with a forceplate mounted on the seat in order to determine factors that could contribute to the formation of decubitus ulcers. All three groups of subjects sat on ROHO and Jay cushions; in addition to sitting on the two cushions, the able-bodied subjects sat on a hard surface. Factors studied were: normal and shear seat forces, the location of the center of mass, and armrest force. The forceplate was under the cushions; therefore, the values reflect average forces over the buttocks and posterior thighs. These factors were compared between disability levels as well as between surface types. Larger, normal, and forward shear forces and a more anterior position of the center of mass were observed with the ROHO cushion. More frequent and larger lateral weight shifts occurred with the Jay cushion. The armrests tended to support from 5 percent of the body weight for quadriplegics to 9 percent for paraplegics. The results suggest that armrests reduce seat forces by carrying some of the body weight.
\end{abstract}

Key words: behavior, cushions, decubitus ulcers, paraplegics, pressure sores, quadriplegics, seating, shear, wheelchairs.

\section{INTRODUCTION}

Pressure sores remain a significant problem for anyone who is confined to a wheelchair. It has been found

Address all correspondence and requests for reprints to: Robert Patterson, $\mathrm{PhD}$, University of Minnesota Hospital, UMHC Box 297, Minneapolis, MN 55455. This work was supported in part by a grant from the St. Paul Ramsey Medical Education and Research Foundation. that some people who fail to relieve pressure from their ischial tuberosities as often as is suggested have no problem with decubitus ulcers $(7,8)$, while others, under the same circumstances, are more apt to develop them. Clues as to why this variability exists may lie in long-term factors such as normal (downward) and shear forces, weight shifts, and cushion type. There is evidence that paraplegics generate more seating shear than able-bodied individuals (1) and that the frequency and intensity of weight displacements correlate with subjective seating comfort $(2,4,6)$. This study investigated the above-mentioned factors on subjects who sat in a wheelchair for 30 minutes.

\section{METHODS}

Experiments were conducted using an Everest \& Jennings Premier powered reclining wheelchair (Everest \& Jennings, Los Angeles, CA). The footrests were removed and the back was kept in a full upright position during the course of the experiments. Subjects' feet were elevated on supports so that the thighs were parallel to the floor. Use of the wheelchair armrests was allowed.

An AMTI force platform (Newton, MA) replaced the standard wheelchair seat. The platform was used to measure normal force, shear force, and the moments around both the lateral and anterior-posterior axes along the surface of the plate. Figure 1 shows the force platform arrangement. The moments were divided by the normal force to determine the anterior-posterior and lateral positions of the center of normal sitting force. Position readings nearer the 


\section{MODIFIED FORCE PLATE ASSEMBLY}

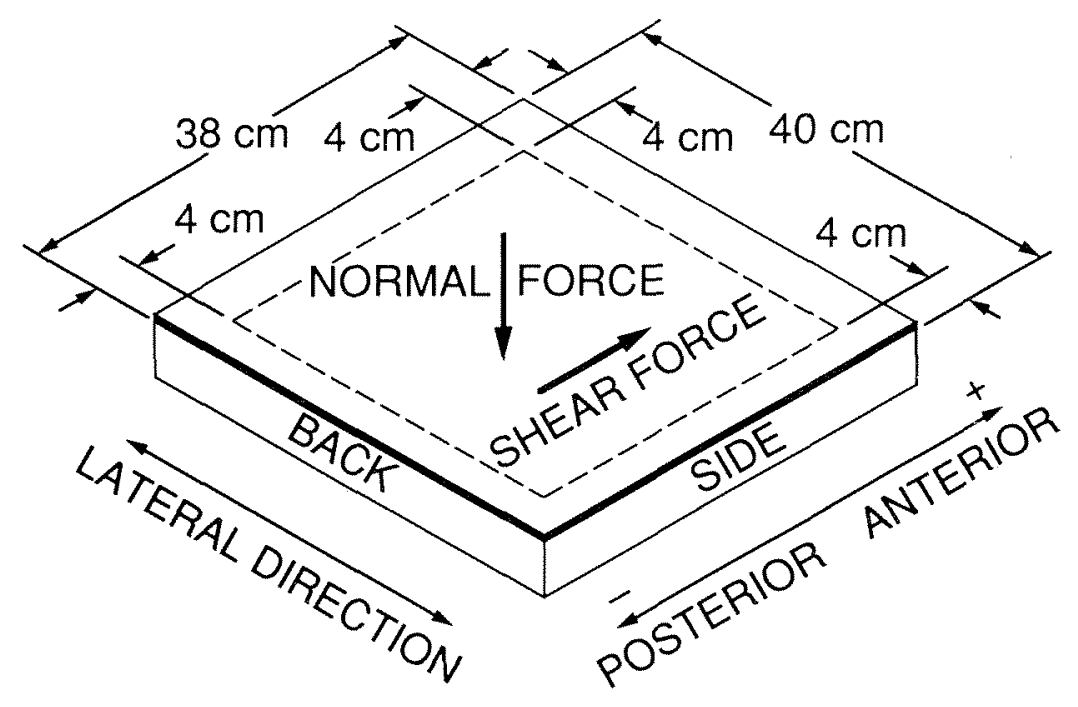

\section{PLATE ASSEMBLY CROSS-SECTION (NOT TO SCALE)}

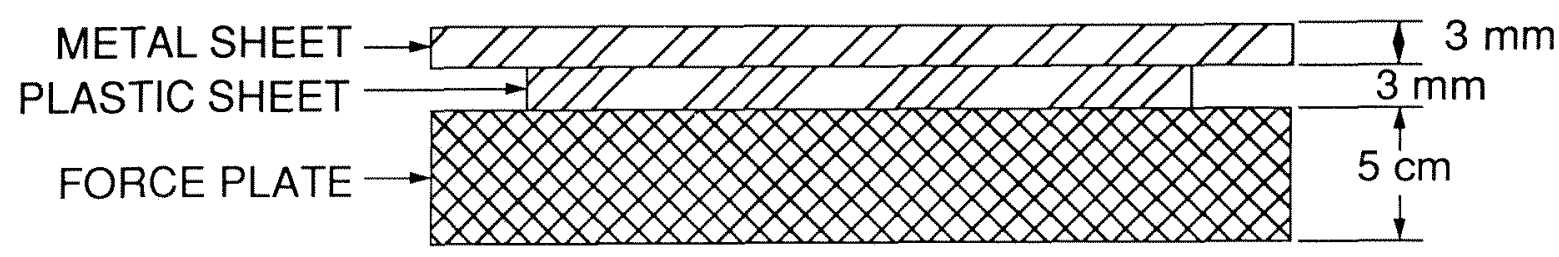

Figure 1.

Modified forceplate with axes and force directions defined.

rear of the plate indicate a greater percentage of force on the ischial tuberosities while readings nearer the front indicate a greater force percentage on the thighs. Normal force is positive downward while shear force and anteriorposterior position are both positive in the anterior direction. Position is zero at the center of the plate.

Due to the thin construction of the plate, there was a problem with obtaining the correct normal force readings along the edges. A modification done to improve accuracy is shown in Figure 1. First, a $3 \mathrm{~mm}$-thick sheet of hard plastic was fastened to the surface of the plate which was cut back $4 \mathrm{~cm}$ from the edges of the plate. A metal sheet of this same thickness but of the same dimensions as the plate surface, was then fastened on top of the plastic sheet. This would redirect normal forces from the edge to the center of the plate. After modification, normal force readings of a $25 \mathrm{~kg}$ weight placed over any portion of the sur- face of the plate differed by less than 5 percent.

Data were collected with an Apple Ile computer (Apple Computer, Cupertino, CA) with an Applied Engineering 12-bit A/D converter (Applied Engineering, Carrollton, TX) through a custom interface box. Strain gauge bridge circuits in the forceplate were excited with 4.5 volts. All resultant analog voltage output signals were run through a two-pole low-pass Butterworth filter with a cutoff frequency of $3.5 \mathrm{~Hz}$ before being digitized. Data were continuously sampled at $10 \mathrm{~Hz}$ throughout the 30-minute testing session.

Three groups of test subjects were used. Six subjects labeled "able-bodied" were apparently healthy young adults with no physical disabilities. Six paraplegics and five quadriplegics made up the other two groups. Disabled subjects with complete sensory loss below the waist were chosen so that movement from seating discomfort would 
Table 1.

Average values of dynamic factors.

\begin{tabular}{llcccccc}
\hline $\begin{array}{c}\text { Subject } \\
\text { Group }\end{array}$ & Seating & $\begin{array}{c}\text { Normal Force } \\
\text { SD }\end{array}$ & $\begin{array}{c}\text { Shear Force } \\
\text { SD (kg) }\end{array}$ & $\begin{array}{c}\text { Lateral Pos. } \\
\text { SD (cm) }\end{array}$ & $\begin{array}{c}\text { A-P Pos. } \\
\text { SD (cm) }\end{array}$ & Zero-Cross & $\begin{array}{c}\text { Arm Force } \\
\text { SD (\% body wt.) }\end{array}$ \\
\hline able-bodied & ROHO & $2.9 \%$ & 0.85 & 0.42 & 1.15 & 13.3 & $9.5 \%$ \\
& Jay & $2.4 \%$ & 1.88 & 0.78 & 0.47 & 19.0 & $8.9 \%$ \\
& Hard & $3.2 \%$ & 1.38 & 0.76 & 1.15 & 18.8 & $9.8 \%$ \\
\multirow{2}{*}{ quad } & ROHO & $3.1 \%$ & 0.76 & 0.34 & 0.27 & 11.3 & $7.7 \%$ \\
& Jay & $3.7 \%$ & 1.13 & 0.49 & 0.40 & 18.0 & $7.5 \%$ \\
para & ROHO & $4.7 \%$ & 1.06 & 0.42 & 0.42 & 16.8 & $11.2 \%$ \\
& Jay & $4.7 \%$ & 0.88 & 0.65 & 0.78 & 31.3 & $10.6 \%$ \\
\hline
\end{tabular}

Hard=subject seated directly on force plate.

$\mathrm{A}-\mathrm{P}=$ anterior-posterior.

not be a factor. Average body weights were $76 \mathrm{~kg}$ for the able-bodied subjects, $66 \mathrm{~kg}$ for the quadriplegics, and 83 $\mathrm{kg}$ for the paraplegics.

The experiments consisted of 30-minute sitting sessions. A 5-second recording was first made with the subjects' arms off the armrests. This data would later be used for normalization and to determine the amount of weight supported by the arms during the long data collection period. Thereafter, the subjects were allowed to use the armrests as they wished. After allowing 10 minutes for the subject to become comfortable, the 30 -minute session was begun. The subjects were instructed to sit normally but were not permitted to remove their feet from the supports.

All three groups of subjects sat on ROHO and Jay cushions; in addition to sitting on the two cushions, the able-bodied subjects sat directly on the forceplate. For subjects sitting directly on the plate, a block of wood $1.4 \mathrm{~cm}$-thick was placed under the plate to keep the subjects at the same relative height in the chair. The ROHO cushion was inflated as stated in the directions supplied by the manufacturer. The order in which the seating surfaces were used by each subject was randomized. Quadriplegic subjects were allowed to use straps for support as needed.

For analysis, the normal force readings were standardized to percent of body weight. The mean and standard deviations were calculated for the normal and outward shear forces as well as for the center of force. The standard deviation was used as an indication of the subjects' activity level while they sat. As a further indicator of lateral weight shift, a count was calculated of times that the weight shifted across the center of the plate. This was labeled in the data as zero-crossings. A $1 \mathrm{~cm}$ "dead zone" was used which had to be crossed before a weight shift would be registered.

To determine the amount of weight supported by the armrests, the weight measurement without armrests was subtracted from the average normal force readings. All the force values are given in kilograms equivalent at normal gravity in order to be easily compared to body weight.

\section{RESULTS}

Data were analyzed using 2-way analysis of variance with 5 percent and 10 percent significance levels. Cushion effects were investigated separately for the ablebodied and two disability classifications, as well as with all subjects grouped together. Additionally, statistical analyses were performed to detect any differences between disability levels. The data were categorized into dynamic and static factors. Dynamic factors reflect movement, and include standard deviation of the force and position readings and lateral zero-crossings. Static factors are those that reflect information about a surface support characteristic or posture of a subject, and include the average value readings.

Dynamic factors gave three statistically significant results: 1) quadriplegics showed larger amplitude lateral movements on the Jay cushion than on the ROHO cushion; 
Table 2.

Differences in the means of static factors.

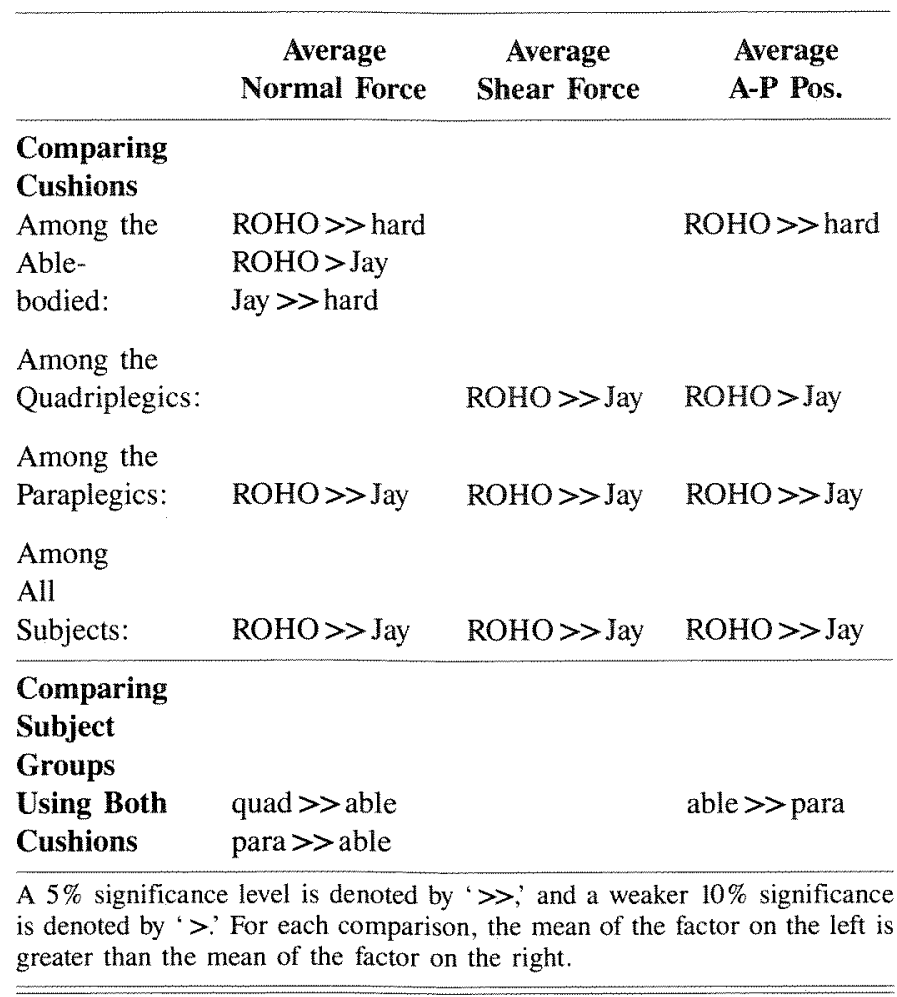

2) paraplegics showed more lateral zero-crossings on the Jay cushion than on the ROHO cushion; and, 3) variation of arm force was largest with paraplegics and least with quadriplegics. Table 1 shows the averaged values of the dynamic factors.

Static factors gave statistically significant differences both between cushion types and disability levels. These differences are shown in Table 2 . With regard to cushion types, subjects sitting on the ROHO cushion tended to generate both greater normal and shear forces, as well as a more anterior center of force, than did subjects sitting on the Jay cushion. With regard to disability levels, the static factor differences between the ROHO and Jay cushions varied the most with paraplegic subjects and the least with quadriplegic subjects. Normal forces were proportionally higher with both quadriplegic and paraplegic subjects than with able-bodied subjects. Less statistically strong was the tendency for the able-bodied subjects to have a more anterior center of force than the paraplegics, while the quadriplegics had an intermediate value. At a significance level of $\mathrm{p}<0.11$, armrest forces applied by paraplegics were larger than those applied by quadriplegics. This force averaged from 8 percent to 9 percent of body weight for the able-bodied subjects and the paraplegics, and approximately 5 percent of body weight for the quadriplegics. Table 3 shows the averaged values of the static factors.

\section{DISCUSSION}

In order to interpret the results, it is helpful to consider the differences between the ROHO and Jay cushion designs. The former is constructed with many pliable air-filled "fingers" projecting upwards, providing a "floating" effect. The Jay cushion is made of a firm, anatomically-contoured, supportive material, covered with gel-filled pouches. The front section of the Jay cushion (which would be situated under the thighs) is built up and has less gel.

Since all measurements were taken on the underside of the cushion, they reflect the average stresses experienced over the buttocks and posterior thighs. These measurements serve to allow relative comparisons between the different test conditions, although peak values on the buttocks may be higher.

Some of the differences measured between cushion types can be attributed to properties of the cushions themselves, rather than from any behavioral differences in the subjects. Increased thigh support affects static factors in two ways. First, leg weight that would normally be carried by the footrests is transferred to the seating surface. Secondly, since this transferred weight is supported by the front of the cushion (under the thighs), the center of normal force is shifted anteriorly. These effects are seen in the data with the normal force and anterior position values, in general, being highest with the ROHO cushion and lowest with the hard surface.

The differing properties of the support surfaces also caused lateral position measurements to measure the subjects' success in shifting weight, rather than the amount of effort used in the attempt. The design of the ROHO cushion decreases the change in recorded position that occurs when a person attempts to shift his or her weight. Because of the compliance of the ROHO, subjects tend to float on the cushion. Lateral shifts in pelvic angle cause a redistribution of air in the cushion, which tends to equilibrate pressures between the ischial tuberosities. This action also shifts pressure off high pressure points onto surrounding areas. With the Jay cushion, there were more frequent and larger amplitude lateral movements of the center of mass. This should result in a greater reduction of pressure under one of the ischial tuberosities, compared 
Table 3.

Average values of static factors.

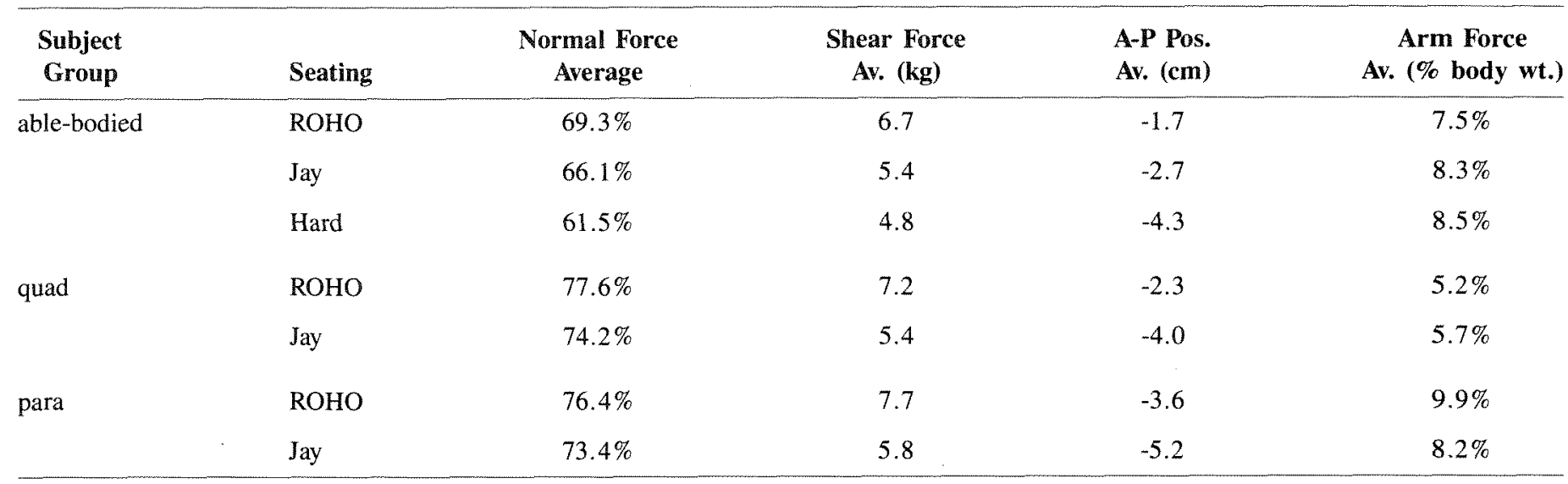

Hard=subject seated directly on force plate.

A-P=anterior-posterior.

to the ROHO, although the pressure would then be transferred to the other tuberosity.

There is also a tendency for greater seating shear with the softer, less stable ROHO cushion than with that of the firmer Jay cushion. This tendency was strongest with the disabled subjects and could be attributed to a desire of the subjects to feel stable, since the shear-deformed cushion should then have less freedom of movement. The fact that shear force was higher with the ROHO cushion than with the Jay cushion would at first seem to favor the Jay cushion, but it must be remembered that it is average shear force, rather than localized shear force, that is being measured. Since more skin surface may be directly supported with the ROHO, the maximum localized shear forces could be the same, or even less, than that of the Jay.

The lower normal force among the able-bodied subjects over that of the paraplegics and the quadriplegics could be due to muscle atrophy reducing the relative leg weight in relation to total body weight. This would cause a greater percentage of total body weight to be supported by the footrests with the able-bodied subjects. This effect is also indicated by the anterior weight placement of the ablebodied subjects.

The results indicate that armrests should be recommended for spinal cord injured patients in wheelchairs. The combined weight of the hand and arm is 10 percent of the body weight (3); therefore, a significant reduction in seating force can be obtained by using armrests, rather than the buttocks, to carry part of the body weight. The paraplegics supported 9 percent of their body weight on the armrests, compared to the quadriplegics, who supported only 5 percent of their body weight. Quadriplegics have weak arm extension and shoulder depressor function. Therefore, they have less ability to laterally shift and lean on their arms. Armrests can also be effective stabilizers, encouraging wheelchair-bound individuals to perform more weight shifts.

In conclusion, the ROHO cushion tends to carry a larger percentage of total body weight and a more anterior center of mass than the other surfaces. Among the handicapped subjects, the ROHO cushion also showed more forward shear force than did the Jay cushion. There was more lateral weight shifting on the Jay cushion than on the ROHO cushion. Also, armrests support a portion of body weight, suggesting that they be used to prevent tissue breakdown problems by reducing seating pressure.

\section{ACKNOWLEDGMENTS}

The authors gratefully acknowledge the efforts of Brenda Roche and the Spinal Cord Society for their help in obtaining test subjects, Jim Barton for his assistance in obtaining equipment, and Nancy Appel for her assistance in data collection.

\section{REFERENCES}

1. Bennet L, Kavner D, Lee BY, Trainor FS, Lewis JM: Skin stress and blood flow in sitting paraplegic patients. Arch Phys Med Rehabil 65(4):186-190, 1984.

2. Coerman R, Rieck A: Eine verfeinerte Methode zur Bestimmung des Komfortgrades von Sitzen. Internationale Zeitschrift fur angewandte Physiologie 20:376-397, 1964. 
Journal of Rehabilitation Research and Development Vol. 28 No. 4 Fall 1991

3. Diffrient N, Tilley AR, Bardagjy JC: Humanscale 1/2/3 Manual. Cambridge: MIT Press, 1974.

4. Dinsdale SM: Decubitus ulcers, role of pressure and friction in causation. Arch Phys Med Rehabil 55:147-152, 1974.

5. Fleischer AG, Rademacher U, Windberg $\mathbf{H J}$ : Individual characteristics of sitting behavior. Ergonomics 30(4):703-709, 1987.

6. Grandjean E, Jenni M, Rhiner A: Eine direkte methode zur- erfassung des komfortgefuhls beim sitzen. Internationale Zeitschrift fur angewandte Physiologie 18:101-106, 1960.

7. Patterson RP, Fisher SV: Pressure and temperature patterns under the ischial tuberosities. Bull Prosthet Res 17(2):5-11, 1980.

8. Patterson RP, Fisher SV: Sitting pressure-time patterns in patients with quadriplegia. Arch Phys Med Rehabil 67(11):812814, 1986. 